\section{Efecto de fertilización orgánica (biol y compost) en el estableci- miento de morera (Morus alba L.)}

\section{The effect of organic fertilization (biol and compost) on the estab- lishment of morera (Morus alba L.)}

\author{
Héctor V. Vásquez y César Maravi²
}

\begin{abstract}
RESUMER
El objetivo del presente estudio fue evaluar el efecto de abono orgánico (biol y compost) en etapa de establecimiento de morera (Morus alba L.) en función de cinco variables agronómicas: (NP) número de plantas $\left(\mathrm{m}^{2}\right)$, (HP) altura de planta (cm), (NM) número de macollos, (PR) profundidad de raíz (cm) y (MS) materia seca kg/ha. A partir del mayor NP, HP y NM, se evaluaron efectos del tiempo de fertilización (95 \% confianza). Para ello se empleó un diseño en bloques completamente al azar (DBCA) en cuatro bloques, diez tratamientos y se hizo la prueba de homogeneidad de varianzas, prueba Duncan. Los resultados de fertilización en cada variable muestran igualdad de varianzas $(p>0,05)$ y normalidad. No se encontraron diferencias estadísticas para HP, NM y MS entre bloques y tratamientos, en cambio para NP hubo diferencia; igual que para PR entre bloques todos evaluadas al noveno mes. El tratamiento $\mathrm{T}_{5}(p<0,05)$, fertilización a razón de $10 \mathrm{t} /$ ha de compost, favoreció significativamente el NP (12/ $\left.{ }^{2}\right)$, NM (72/planta) y HP $(104 \mathrm{~cm})$; con respecto al tiempo de fertilización NP (56 \%) y NM (10\%) fueron significativos y HP (93\%) resultó altamente significativa. El tratamiento $\mathrm{T}_{4}$, compost a razón de $5 \mathrm{t} / \mathrm{ha}$, fue el que generó mayor profundidad de raíz $(60 \mathrm{~cm})$. Producción de MS 15 \% (197,22 kg/ha) tratamiento $\mathrm{T}_{9}(10 \mathrm{t} / \mathrm{ha}$ biol: $5 \mathrm{t} /$ ha compost). Se concluye que compost a razón de $10 \mathrm{t} /$ ha actúa como fertilizante, da mejor crecimiento en etapa de establecimiento de morera, permitiendo disponer mayor número de macollos y plantas $\left(\mathrm{m}^{2}\right)$.
\end{abstract}

\section{Phlabras Clave}

biol $\cdot$ compost $\cdot$ establecimiento $\cdot$ morera

\section{ABSTRACT}

The present study evaluater the effect of organic fertilizer (biological compost) on the establishment staget plant height $(\mathrm{cm})$ mulberry (Morus alba L.). We examine five agricultural variables: (NP) number of plants $\left(\mathrm{m}^{2}\right),(\mathrm{HP}),(\mathrm{NM})$ of number tillers (PR) root depth (cm) and (MS) kg dry biomass / ha. Senior NP, HP, and NM fertilization effects of time (95\% confidence) were evaluated. This requires a design randomized complete block DBCA was used in four blocks, ten treatments; the test of homogeneity of variance, Duncan test was made. The results of fertilization in each variable show equal variances ( $\mathrm{p}$-value> 0.05) and a normal distribution. There no statistical differences for HP, NM and MS between blocks and treatments were found, while for NP was no difference. For PR between blocks all were evaluated during the ninth month. The $\mathrm{T}_{5}(\mathrm{p}$ $<0.05$, Duncan test), fertilization at $10 \mathrm{t} /$ ha of compost, treatment significantly favored the NP $\left(12 / \mathrm{m}^{2}\right), \mathrm{NM}(72 /$ plant $)$ and HP $(104 \mathrm{~cm})$; with respect to time of fertilization NP (56\%) and NM (10\%) were significant and HP (93\%) was highly significant. $\mathrm{T}_{4}$ treatment, composting at $5 \mathrm{t} / \mathrm{ha}$ was generated deeper roots $(60 \mathrm{~cm}) .15 \% \mathrm{DM}$ yield (197.22 kg/ha) treatment $\mathrm{T}_{9}(10 \mathrm{t} / \mathrm{ha}$ biol 5 $\mathrm{t} /$ ha compost). It is concluded that compost at the rate of $10 \mathrm{t} / \mathrm{ha}$ acts as fertilizer gives better growth during the establishment stage of mulberry, allowing higher planting dersity having greater number of macollos and plants $\left(\mathrm{m}^{2}\right)$.

\section{INTRODUCCIÓN}

La búsqueda de especies con potencial para alimentación animal, en países de América Latina, ha llevado al estudio de leñosas nativas e introducidas que no se utilizan de manera convencional en los sistemas de producción noroeste. En el Perú, y en la región Amazonas, la

\section{KESWORDS}

biol $\cdot$ compost $\cdot$ establishment $\cdot$ morera

'Ingeniero Zootecnista. Investigador del IGBI y docente FIZAB-UNTRM. Correo Electrónico: hvasquez@untrm.edu.pe
'Ingeniero Zootecnista. Investigador del IGBI y docente FIZAB-UNTRM. Correo Electrónico: cesar.maravi@untrm.edu.pe 
su producción por unidad de área (Martín, et al., 2000), así como la necesidad de reponer, al suelo, el nitrógeno extraído por las plantas (ElizondoSalazar, 2010). Para obtener rendimiento de forraje con adecuado nivel de proteína, sin comprometer reservas del suelo, se requiere de una alta dosis de fertilizante químico, lo que incrementa los costos de producción y el riesgo de contaminación ambiental (Elizondo-Salazar, 2007). La utilización de fertilizantes orgánicos (biol y compost) contribuye a una mayor productividad de pasturas, en comparación con los fertilizantes inorgánicos, por el alto efecto contaminante, pérdida progresiva de la fertilidad del suelo, alteración y muerte de microorganismos (Cifuentes y Sohn, 1998). La eficiencia de cultivos que utilizan $\mathrm{N}_{2}$, presente en este tipo de abonos, depende de diversos factores como suelo, planta, clima y manejo (Cifuentes y Sohn, 1998). Sin embargo, se sabe poco sobre la utilización y efecto del abono orgánico en cultivos de alto rendimiento forrajero, como en el establecimiento de morera.

Las principales características de cultivo de pastos, en la región Amazonas, son producidas de manera extensiva $\mathrm{y}$, en consecuencia, bajo rendimiento productivo en carne y leche, lo que se refleja en bajos ingresos y una baja calidad de vida. Las raciones diarias de alimento natural de pastos $\mathrm{y}$ forrajes para ganado deben proveer cantidades adecuadas de nutrientes para el crecimiento, mantenimiento corporal, preñez y producción (ganancia diaria de peso) que otorguen los requerimientos necesarios en energía, proteína, minerales, vitaminas y agua (Isaza, 2006).

El cultivo de morera se introdujo en Europa, en el siglo VI, a través del gusano de seda, luego se distribuyó por toda la mitad sur europea (Parque Zoológico y Jardín Botánico de Jerez). El amplio rango de distribución de morera ha permitido que la planta presente gran capacidad de adaptación a diversas condiciones climáticas. Reportan temperaturas entre 13 y $38{ }^{\circ} \mathrm{C}$, con un rango óptimo entre 24 y $28{ }^{\circ} \mathrm{C}$; además de niveles de precipitaciones entre 600 y $2500 \mathrm{~mm}$ (García, et al., 2006). Por eso, en América se extiende desde EE.UU. hasta Argentina, e incluye México, varios países de Centroamérica, el Caribe, Brasil, Colombia y Perú (Manterola, 2000). Realmente el descubrimiento de esta especie como recurso forrajero alternativo se hizo en Costa Rica, por los años ochenta, donde un agricultor tenía producción de gusano de seda y al no poder continuar con ello, optó por ofrecer el forraje a sus cabras, obtuvo excelentes resultados y decidió hacer pública su experiencia, al Centro Agronómico Tropical de Investigación y Enseñanza (CATIE, Costa Rica). A partir de esta experiencia, se investigó en distintas zonas geográficas (Okamoto et al., 2008). Esta especie forrajera muestra excelentes características de palatabilidad y consumo, tanto en ganado bovino (Elizondo-Salazar, 2007) como caprino (Boschini-Figueroa, 2002; Elizondo-Salazar, 2007). Además, presenta gran versatilidad agronómica, excelente rendimiento de biomasa y calidad nutritiva (Boschini-Figueroa, 2005). Sin embargo, en algunos países, se utiliza como árbol frutal, ornamental (Sánchez, 2002) y árbol de sombra (Medina et al., 2012). Por otra parte, su uso como forraje ha demostrado un gran potencial, por su calidad y producción de follaje, las características organolépticas y el alto consumo animal (Benavides, 1999).

La propagación de esta especie es asexual (estacas), y constituye una forma fácil y rápida de conservar características de la planta madre (Sánchez, 2002). La propagación a nivel de vivero se inicia con el plantado de estacas en sustratos con alto contenido de materia orgánica, como compostajes, que constituyen una fuente de nutrientes de liberación lenta, favorable para los requerimientos de la planta (Borges et al.,2014).

El presente trabajo de investigación tuvo como objetivo, evaluar el efecto de dos abonos orgánicos (biol y compost) en la etapa de establecimiento de morera (Morus alba L.) en la estación experimental Agua Dulce de la UNTRM, localizada en Yambrasbamba, provincia Bongará, mediante la evaluación de niveles de fertilización orgánica e instalación de pasturas, con morera en función de diversas variables agronómicas. Asimismo, determinar la producción de materia seca, bajo el efecto del fertilizante orgánico biol y compost durante el tiempo de crecimiento de morera, que permitirá mejorar calidad nutricional en alimentación del ganado vacuno y por consecuencia rendimiento en carne y leche. Asimismo, permitir el incremento de ingresos del ganadero y mejorar la producción pecuaria con el uso de fertilizantes orgánicos que aumente la producción de forraje y mejore las condiciones ambientales.

\section{MATERIALES Y MÉTODOS Clima, suelo y altitud}

La investigación se realizó en la estación experimental Bongará de la UNTRM, en el período comprendido entre los meses de enero y setiembre del 2015. Durante esta etapa, se registraron $366 \mathrm{~mm}$ a $800 \mathrm{~mm}$ de precipitación y la temperatura media fue de $18 \pm 3{ }^{\circ} \mathrm{C}$. El suelo presenta una topografía 
accidentada y se clasifica como franco arcilloso (Font et al., 2002; Toledo y Schultze-Kraft, 1982).

\section{Plantación}

Se utilizaron propágulos del semillero de la estación experimental de Agua Dulce, con una edad de tres semanas. Estas se colocaron de forma vertical con respecto al suelo, dejando al menos una yema bajo tierra. Se realizaron labores de limpieza para mantener el área libre de plantas adventicias. Se aplicó una fertilización inicial y, a cada mes de plantación, Biol proveniente de biodigestores instalados en la estación experimental y compost elaborado de manera artesanal. El abono inorgánico fue obtenido de casas comerciales de Chachapoyas.

Para la evaluación del efecto de fertilización orgánica en el establecimiento de morera (Morus alba L.), en el noveno mes, se evaluó el número de plantas $\left(\mathrm{m}^{2}\right)$, altura de planta $(\mathrm{cm})$, número de macollos, profundidad de raíz y producción de materia seca.

El experimento se condujo en un Diseño en Bloques Completamente al Azar (DBCA). El criterio de bloqueo fue la pendiente, con lo cual se controló un factor que se esperaba tenga efecto significativo en la variable respuesta y fueron evaluados diez tratamientos incluido el testigo.

Tabla 1. Diseño experimental

\begin{tabular}{ll}
\hline \multicolumn{1}{c}{ Tratamientos (t/ha) } \\
\hline $\mathbf{T}_{\mathbf{1}}$ & Testigo \\
$\mathbf{T}_{\mathbf{2}}$ & $5 \mathrm{Biol}$ \\
$\mathbf{T}_{3}$ & $10 \mathrm{Biol}$ \\
$\mathbf{T}_{\mathbf{4}}$ & 5 Compost \\
$\mathbf{T}_{5}$ & 10 Compost \\
$\mathbf{T}_{6}$ & NPK \\
$\mathbf{T}_{7}$ & 5 Biol $: 5$ Compost \\
$\mathbf{T}_{8}$ & 5 Biol $: 10$ Compost \\
$\mathbf{T}_{9}$ & 10 Biol $: 5$ Compost \\
$\mathbf{T}_{10}$ & 10 Biol : 10 Compost \\
\hline
\end{tabular}

\section{Procedimiento}

a) Número de plantas $\left(\mathrm{m}^{2}\right)$

Para determinar el número de plantas, se usó un metro cuadrado de madera y se contaron plantas que estaban dentro de él. Se realizaron cuatro repeticiones por cada subparcela y luego se obtuvo el promedio final.

\section{b) Altura de planta (cm)}

Se muestrearon cinco plantas seleccionadas al azar en cada subparcela y fueron evaluadas desde el primer mes hasta el noveno mes. La medición se hizo desde el suelo hasta el punto más alto de la planta, sin estirar hojas ni contar la inflorescencia. Se promediaron mediciones tomadas de dos plantas grandes, dos medianas y una planta pequeña.

\section{c) Número de macollos}

Se realizó el conteo de número de plantas en área de $1 \mathrm{~m}^{2}$ y se contaron número de macollos de cada planta de morera.

\section{d) Profundidad de raíz $(\mathrm{cm})$}

En el primer y segundo corte, se tomaron tres plantas al azar de cada parcela y se midieron $(\mathrm{cm})$ desde el cuello de la raíz hasta el meristemo terminal de la raíz más larga.

\section{e) Producción de materia seca (kg/ha)}

Se registraron datos de peso fresco de muestra $\left(\mathrm{g} / \mathrm{m}^{2}\right)$, peso fresco de submuestra $\left(\mathrm{g} / \mathrm{m}^{2}\right)$ y peso seco de muestra (g). A partir de esta, se tomaron submuestras de $250 \mathrm{~g}$ para determinación de materia seca (MS). Las submuestras se colocaron en bolsas de papel, rotuladas por fecha, especie y tratamiento. Fueron secadas en estufa a temperatura de $60{ }^{\circ} \mathrm{C}$ a peso constante y luego fueron pesadas a temperatura ambiente.

El peso de materia seca se determinó aplicando la fórmula siguiente :

$$
M S / m^{2}=\frac{P F X p s}{p f}
$$

Donde:

PF : Peso fresco de la muestra $\left(\mathrm{g} / \mathrm{m}^{2}\right)$

pf : Peso fresco de la submuestra (g)

ps $\quad$ :Peso seco de la submuestra (g)

Además, se realizaron evaluaciones del tiempo de establecimiento frente a las variables de número de plantas, número de macollos por planta macollos y altura de planta.

Para el análisis estadístico, se empleó el SPSS (SmartViewer 20.0, Microsoft), realizado de homogeneidad de varianzas y para comparación de medias la prueba Duncan.

\section{RESULTADOS}

En la tabla 1, se muestran valores promedios de 5 variables en estudio: altura $(\mathrm{cm})$, número de plantas $\left(\mathrm{m}^{2}\right)$, número de macollos, profundidad de raíz $(\mathrm{cm})$ y producción de materia seca de diferentes tratamientos evaluados, al noveno mes de establecida la morera (Morus alba L.)

En base a la prueba de comparación múltiple, no se observó efecto significativo $(p<0,05$, prueba Duncan) para todas las otras variables y para altura de plantas de morera $(\mathrm{cm})$ entre fertilizantes.

En base a la prueba de comparaciones múltiples, específicamente la prueba de Duncan se observó que la fertilización que obtuvo menor altura de planta (66 
cm) fue el T1 (testigo). La que obtuvo mayor altura de planta $(104 \mathrm{~cm})$ fue el tratamiento T5 (fertilización con $10 \mathrm{t} /$ ha de compost).

Asimismo, respecto al número de plantas $\left(\mathrm{m}^{2}\right)$, no se observó efecto significativo entre fertilizantes (tratamientos $\mathrm{T}_{2}, \mathrm{~T}_{5} \mathrm{y} \mathrm{T}_{9}$ ). Es decir, la fertilización a razón de $5 \mathrm{t}$ /ha de biol, $10 \mathrm{t} / \mathrm{ha}$ de compost, 10 t/ha de biol y 5 t/ha de compost. Se encontraron medias de 12,25, 11,75 y 11,50 respectivamente. El recomendado será el tratamiento 5 (fertilización a razón de 10 t/ha de compost), por los bajos costos de producción. A pesar de que la fertilización a razón de 5 t/ha de biol dio mayor número de plantas $\left(\mathrm{m}^{2}\right)$. El menor número de plantas al fertilizar fue a razón de 10 t/ha de biol y $5 \mathrm{t} /$ ha de compost.

Tabla 2. Promedios evaluados de cinco variables al noveno mes de establecida la morera por tratamiento

\begin{tabular}{cccccc}
\hline $\begin{array}{c}\text { Trat. } \\
(\mathbf{t} / \mathbf{h a})\end{array}$ & $\begin{array}{c}\mathrm{N}^{\circ} \text { de } \\
\text { plantas }\left(\mathrm{m}^{2}\right)\end{array}$ & $\begin{array}{c}\text { Altura de } \\
\text { planta }(\mathrm{cm})\end{array}$ & $\mathrm{N}^{\circ}$ de macollos & $\begin{array}{c}\text { Profundidad } \\
\text { de raíz }(\mathrm{cm})\end{array}$ & $\begin{array}{c}\text { Materia seca } \\
(\mathrm{kg} / \mathrm{ha})\end{array}$ \\
\hline $\mathbf{T}_{\mathbf{1}}$ & $09,50^{\mathrm{ab}}$ & $66,10^{\mathrm{a}}$ & $33,00^{\mathrm{b}}$ & $41,47^{\mathrm{ab}}$ & $55,94^{\mathrm{b}}$ \\
$\mathbf{T}_{\mathbf{2}}$ & $12,25^{\mathrm{a}}$ & $70,40^{\mathrm{a}}$ & $51,25^{\mathrm{ab}}$ & $33,84 \mathrm{~b}$ & $83,18^{\mathrm{ab}}$ \\
$\mathbf{T}_{\mathbf{3}}$ & $09,75^{\mathrm{ab}}$ & $91,50^{\mathrm{a}}$ & $32,75^{\mathrm{b}}$ & $38,34^{\mathrm{ab}}$ & $111,80^{\mathrm{ab}}$ \\
$\mathbf{T}_{\mathbf{4}}$ & $09,50^{\mathrm{ab}}$ & $71,70^{\mathrm{a}}$ & $38,00^{\mathrm{b}}$ & $59,71 \mathrm{a}$ & $75,35^{\mathrm{ab}}$ \\
$\mathbf{T}_{\mathbf{5}}$ & $11,75^{\mathrm{a}}$ & $104,00^{\mathrm{a}}$ & $71,50^{\mathrm{a}}$ & $29,42^{\mathrm{b}}$ & $159,45^{\mathrm{ab}}$ \\
$\mathbf{T}_{\mathbf{6}}$ & $07,25^{\mathrm{b}}$ & $106,50^{\mathrm{a}}$ & $30,50^{\mathrm{b}}$ & $45,30^{\mathrm{ab}}$ & $184,17^{\mathrm{ab}}$ \\
$\mathbf{T}_{\mathbf{7}}$ & $09,25^{\mathrm{ab}}$ & $78,65^{\mathrm{a}}$ & $41,25^{\mathrm{b}}$ & $33,066^{\mathrm{b}}$ & $65,77^{\mathrm{b}}$ \\
$\mathbf{T}_{\mathbf{8}}$ & $09,75^{\mathrm{ab}}$ & $84,20^{\mathrm{a}}$ & $39,75^{\mathrm{b}}$ & $35,27^{\mathrm{ab}}$ & $76,90^{\mathrm{ab}}$ \\
$\mathbf{T}_{\mathbf{9}}$ & $11,50 \mathrm{a}$ & $103,35^{\mathrm{a}}$ & $49,25^{\mathrm{ab}}$ & $36,83^{\mathrm{ab}}$ & $* 197,22 \mathrm{a}$ \\
$\mathbf{T}_{\mathbf{1 0}}$ & $09,75^{\mathrm{ab}}$ & $78,20^{\mathrm{a}}$ & $44,75^{\mathrm{ab}}$ & $34,50^{\mathrm{ab}}$ & $67,52 \mathrm{~b}$ \\
\hline
\end{tabular}

* De 197,22 kg de materia en base húmeda, se obtuvo $15 \%$ de materia seca

${ }^{\mathrm{ab}}$ Medias con letras distintas dentro de la misma columna son significativamente diferentes $(\mathrm{p}<0.05)$, prueba Duncan

La figura 1 muestra los mejores niveles observados durante el tiempo de nueve meses de fertilización respecto al número de plantas $\left(\mathrm{m}^{2}\right)$. Por ello, la fertilización a razón de $5 \mathrm{t} / \mathrm{ha}$ de biol $\left(\mathrm{T}_{2}\right)$, gráficamente, tiene tendencia polinómica para un $\mathrm{R}^{2}=$ 0,9755 y obtuvo valores de $y=0,1339 x^{2}-1,6643 x$ $+16,502$. Seguido del $T_{5}$ (10 t/ha compost) con tendencia polinómica para un $\mathrm{R}^{2}=0,9944$ obtuvo valores de $y=0,067 x^{2}-1,0571 x+15,776$. Y por último el $\mathrm{T}_{9}(10 \mathrm{t} /$ ha Biol: $5 \mathrm{t} /$ ha compost $)$ con tendencia de potencia para un $\mathrm{R}^{2}=0,9167$ obtuvo valores de $\mathrm{y}=14,833 \mathrm{x}^{-0,12}$. Esto se corresponde con el análisis de varianza (tabla 2) un 56 \% del número de plantas de morera al $95 \%$ de confianza dependen del tiempo de fertilización.

En base a la prueba de comparación múltiple, no se observó efecto significativo en el tratamiento 5. Es decir, la fertilización a razón de 10 t/ha compost es la que obtuvo mayor número de macollos (72/planta). Con respecto a los otros tratamientos, hubo diferencia significativa. Por eso, se recomienda el tratamiento 5. Estos resultados sugieren, en base a las otras dos variables anteriores altura de planta y número de plantas, que se obtienen mejores resultados con este tratamiento. En cuanto a profundidad de raíz, no hubo diferencia significativa para el tratamiento 4.

El mayor contendido de materia seca $(197,22 \mathrm{~kg})$ lo consiguió el tratamiento $9\left(\mathrm{~T}_{9}\right)$; es decir, cuando se aplicó 10 t/ha de Biol + 10 t/ha de compost.
Para este tratamiento se encontró diferencia no significativa. En cambio, cuando se aplicó 10 t/ha compost $\left(\mathrm{T}_{5}\right)$ y NPK $\left(\mathrm{T}_{6}\right)$, hubo diferencia estadística y se econtraron valores menores de 159,45 y 184,17 de MS respectivamente.

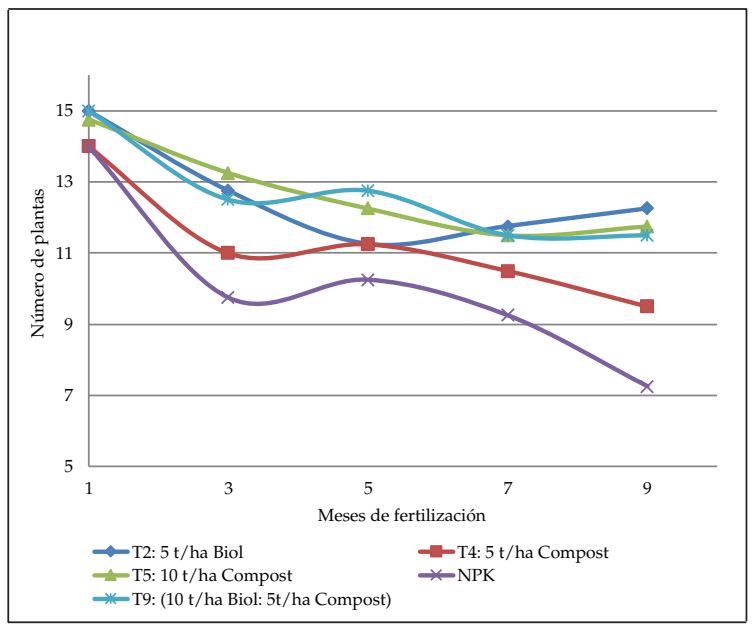

Figura 1. Número de plantas de morera $\left(\mathrm{m}^{2}\right)$

Del mismo modo, la figura 2 muestra atura promedio de plantas $(\mathrm{cm})$ de morera tras nueve meses de fertilización. El $\mathrm{T}_{5}$ y $_{9}$ gráficamente ambas con tendencia de potencia de valores muy cercanos para $R^{2}=0,975$ se obtuvo valores de $y=26,518 x^{0,6037}$. 
Tabla 3. ANVA para número de plantas, número de macollos y altura de plantas de morera (Morus alba L.) con respecto al tiempo de fertilización

\begin{tabular}{lcccccccc}
\hline $\begin{array}{l}\text { Variables de } \\
\text { plantas de } \\
\text { morera }\end{array}$ & $\begin{array}{c}\text { Grados } \\
\text { de } \\
\text { libertad }\end{array}$ & $\begin{array}{c}\text { Promedio } \\
\text { de los } \\
\text { cuadrados }\end{array}$ & $\begin{array}{c}\text { Coeficiente de } \\
\text { correlación } \\
\text { múltiple }\end{array}$ & $\begin{array}{c}\text { Coeficiente de } \\
\text { determinación } \\
\mathbf{R}^{\wedge 2}\end{array}$ & Error típico & $\boldsymbol{F}$ & $\begin{array}{c}\text { Valor crítico } \\
\text { de } \boldsymbol{F}\end{array}$ \\
\hline $\mathbf{n}^{\circ}$ plantas & 1 & 6,02 & 0,75 & 0,56 & 0,82 & 9,03 & 0,02 & $*$ \\
$\mathbf{n}^{\circ}$ macollos & 1 & 24,07 & 0,31 & 0,095 & 7,94 & 0,75 & 0,42 & $*$ \\
Altura plantas & 1 & 6106,06 & 0,97 & 0,933 & 5,71 & 96,81 & 0,000238 & $* *$ \\
\hline
\end{tabular}

* Significativo

** Altamente significativo

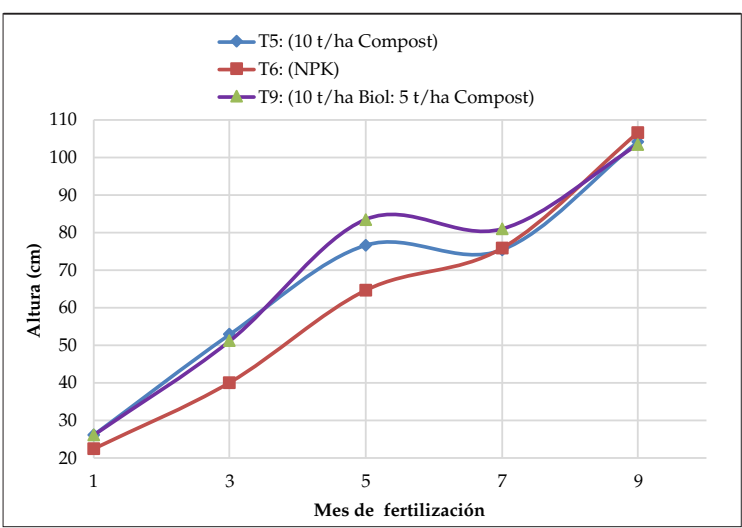

Figura 2. Altura media de plantas $(\mathrm{cm})$ de morera según meses de fertilización

El tratamiento $\mathrm{T}_{6}$ (NPK) ha tenido un crecimiento polinómico por encima de los otros dos tratamientos. Luego de la relación de un análisis de varianza, se encontró que el $93 \%$ de altura de plantas depende del tiempo de fertilización a razón de 10 $\mathrm{t} /$ ha de compost (tabla 3 ).

Esto indica que la morera en su condición de no leguminosa, presenta gran dependencia de la fertilización química u orgánica cuando su biomasa es usada intensivamente en los sistemas de alimentación (Martín et al., 2002; Benavides, 1999).

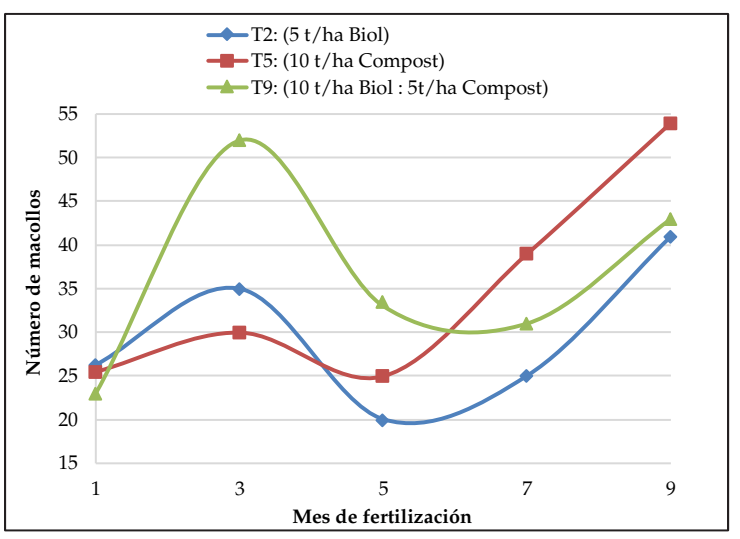

Figura 3. Número de macollos en morera según meses de fertilización

La figura 3 muestra el número de macollos a través del tiempo en la que el $T_{5}(10 \mathrm{t} / \mathrm{ha}$ de compost) gráficamente tiene tendencia polinómica para un $\mathrm{R}^{2}=0,9292$ se obtuvo valores de $\mathrm{y}=0,7143 \mathrm{x}^{2}-$
$3,8429 \mathrm{x}+30,343$; en cambio, los $\mathrm{T}_{2} \mathrm{y} \mathrm{T}_{9}$ obtuvieron menor número de macollos a pesar que en un inicio tiene tendencia de aumento. Según el análisis de varianza, sólo el $10 \%$ del desarrollo del macollo por planta de morera dependen del tipo de fertilización (tabla 3).

\section{DISCUSIÓN}

En la mayoría de predios ganaderos de la región Amazonas y del país, existe ausencia de pasturas mejoradas. Además las especies forrajeras aprovechadas al corte, pastos y forrajes son poco productivas y no aportan nutrientes necesarios que el animal necesita para la producción de carne o leche (Martín et al., 2007). Por eso hubo la necesidad de realizar el establecimiento y manejo de pastos de corte con morera (Morus alba L.) en la región Amazonas. El aporte de materia orgánica al suelo, durante el establecimiento de las plantaciones de morera, proporciona una ventaja significativa en la respuesta productiva del arbusto forrajero. El análisis estadístico mostró no significancia entre tiempo de fertilización NP y NM, en cambio diferencia estadística para HP. Estas Variaciones en el crecimiento demuestran dependencia al abono orgánico, que influencia directamente por el incremento de niveles de fertilización (Pentón, 2007).

La morera es una especie vegetal que requiere un suministro adecuado de nutrientes al suelo para poder expresar su alto potencial productivo. En este sentido, en base de la teoría del balance de materia orgánica (biol y compost), se ha demostrado que las variaciones de crecimiento y respuesta a tamaño, NP, PR y NM, dependen del porcentaje de fertilización (García et al., 2006).

Es decir, NP concuerda con los resultados encontrados por Noda et al. (2007), en la diferencia no significativa para un promedio de 12,50 plan$\mathrm{tas} / \mathrm{m}^{2}$. Del mismo modo, la mayor densidad que planteó Páez (1991), que la luz influye en el crecimiento y desarrollo de las plantas de varias maneras, según su calidad, intensidad y duración. El uso de altas densidades de siembra acarrea problemas relacionados con la competencia dentro del cultivo. Se infiere que las densidades muy bajas o muy altas influyen en el desarrollo final 
del ciclo de la planta. Por tal razón, es importante tener en cuenta el área vital de la morera.

La propiedad del abono orgánico de ser asimilable por las plantas, a través de su sistema radical o por el tejido foliar, y participación en el metabolismo al activar el proceso fotosintético. A ello se debió el efecto obtenido en cada una de las variables en estudio. También Huelva et al. (2004) obtuvieron un incremento foliar que posibilitó una mayor captación de energía radiante y más acumulación fotosintética, ya que cuando aumenta la disponibilidad de material orgánico en el suelo, también aumenta la absorción radicular (Hurtado et al., 2012; Monter, 2012). Se presupone que la fertilización orgánica a razón de 5 t/ha de compost influencia en la mayor profundidad de la raíz en el tratamiento 4 con respecto a los otros tratamientos se observa una menor profundidad de raíz. Sin embargo, es importante recordar que el tipo de suelo, grado de inclinación del terreno, pueden ser el resultado de estas variaciones de la PR.

En cualquier caso, los niveles de fertilización a razón de $10 \mathrm{t} /$ ha de compost se obtienen mayor $\mathrm{NM}, \mathrm{NP}, \mathrm{HP}$ y a razón de $5 \mathrm{t} /$ ha de compost se obtiene mayor PR.

De manera general se pudo apreciar la evolución, en 9 meses, de tres variables productivas de la morera con respecto a la fertilización orgánica (Pentón, 2007). En este sentido, Figueroa et al. (2001) informaron que el abono orgánico aplicados al suelo permiten un aumento de la fertilidad del suelo, mejoran sus propiedades físicas y facilitan un mejor aprovechamiento de algunos macronutrientes, todos estos aspectos repercutieron en la producción de materia seca (15 \% MS; 197,22 $\mathrm{kg}$ ). Es decir $10 \mathrm{t} / \mathrm{ha}$ de Biol $+5 \mathrm{t} / \mathrm{ha}$ de compost. Aunque al suelo se le aplicó NPK, no hubo una respuesta en el número de plantas, a pesar de indicar lo contrario, además de obtener mayor número de plantas, esto debido quizás a la inmovilización como producto del $\mathrm{pH}$ ácido que posee este suelo; resultados que coinciden con los hallados por Gallardo et al. (2001).

Sin embargo, fue notorio el efecto del compost, sobre todo en el número de macollos (72/ planta) y número de plantas $\left(12,50 \mathrm{~m}^{2}\right)$.

Noda et al. (2007) obtuvieron resultados similares cuando evaluaron número de plantas/ha.

Según Pentón (2007), la utilización de fertilizantes orgánicos en la actualidad es la mejor opción para la productividad de pasto de morera, en comparación con el alto efecto contaminante, pérdida progresiva de fertilidad del suelo, alteración y muerte de microorganismos del suelo por aplicación masiva de fertilizantes inorgánicos o químicos. Asimismo, costos de producción del compost es más favorable seguidamente del producto secundario que se obtiene a través del biogás mediante biodigestores (biol), este último el costo de instalación no justifica costos de producción como abono orgánico para este estudio.

\section{CONCLUSIONES}

El estudio demuestra que morera (Morus alba L.) fertilizada con abono orgánico a razón de 10 t/ha de compost $\left(\mathrm{T}_{5}\right)$ favorece la producción en la obtención de mayor número de plantas $12 / \mathrm{m}^{2}$, mayor altura de plantas $(104 \mathrm{~cm})$ y número de macollos (72/planta).

El $56 \%, 93 \%$ y $10 \%$ del NP, HP y NM respectivamente dependen del tiempo de fertilización. Esta hipótesis se apoya en que menores densidades de plantación $/ \mathrm{m}^{2}$ influyen significativamente en el buen establecimiento de morera.

La mayor profundidad radicular se obtuvo al aplicarse fertilización a razón de 5 t/ha de compost $\left(\mathrm{T}_{4}\right)$. Su elección como abono orgánico es, el bajo costo y producción existente en la zona ganadera del estudio.

La mayor producción de materia seca se encontró cuando se fertilizó con biol y compost a razón de 5 y $10 \mathrm{t} /$ ha respectivamente $\left(T_{9}\right)$.

Se debe realizar evaluación bromatológica y de rendimiento de la morera.

\section{REFERENCIAS BIBLIOGRÁFICAS}

Benavides, J. E. (1999). Utilización de la morera en sistemas de producción animal. FAO Animal Production and Health Paper, 275-294.

Boschini-Figueroa, C. (2005). Nutrientes digeribles, energía neta y fracciones proteicas de la morera (Morus alba) aprovechables en vacas lecheras. Agronomía Mesoamericana, 17 (2), 141-150.

Borges, J. A., Barrios, M., Chávez, A., y Avendaño, R. (2014). Efecto de la fertilización foliar con humus de lombriz Líquido Durante el aviveramiento de la morera (Morus alba L.). Bioagro, 26 (3), 159-164.

Cifuentes Correa, C. A., \& Sohn, K. W. (1998). Manual técnico de sericultura: cultivo de la morera y cría del gusano de seda en el trópico.

Elizondo-Salazar, J. A., \& Boschini-Figueroa, C. (2002). Producción de forraje con maíz criollo y maíz híbrido. Agronomía mesoamericana, 13(1), 13-17.

Elizondo-Salazar, J. A. (2007). Producción y calidad de la biomasa de morera (Morus alba) fertilizada con diferentes abonos. Agronomía Mesoamericana, 18 (2), 255-261.

Elizondo-Salazar, J. A. (2010). Respuesta de la more- 
ra (Morus alba) a niveles crecientes de nitrógeno orgánico. Pastos y Forrajes, 33(3), 0-0.

Figueroa, R.; Delgado, M.; Berumen, S.; Salazar, E. \& Vázquez, C. (2001). Rendimiento y calidad de forrajes en alfalfa (Medicago sativa) con fertirrigación y ácidos húmicos. Resúmenes. XV Congreso Latinoamericano y V Cubano de la Ciencia del Suelo. Comisión V. Fertilidad y Nutrición. Boletín $\mathrm{n}^{\circ}$. 4 Centro de Convenciones Plaza América. Varadero, Cuba.p. 121.

Font, L., Calero, B., \& Del Castillo, A. (2002). Estado microbiológico del suelo, base del manejo integral de un agroecosistema citrícola. LEISA. Rev. de Agroecología, 18(3), 21-22.

García, D. E., Medina, M. G., Domínguez, C., Baldizán, A., Humbría, J., \& Cova, L. (2006). Evaluación química de especies no leguminosas con potencial forrajero en el estado Trujillo, Venezuela. Zootecnia Trop, 24 (4), 401.

Gallardo, F., Vistoso, E., Pino, M., \& Borie, F. (2001). Absorción de fósforo de cultivares de cebada y trigo crecidos en solución nutritiva. Resúmenes. XV Congreso Latinoamericano y V Cubano de la Ciencia del Suelo. Boletín de la Sociedad Cubana de la Ciencia del Suelo, (4), 130.

Isaza, C. J. J. (2006). Evaluación nutricional y agronómica de Morus alba L y Sambucus nigra L y su utilización en alimentación de rumiantes y monogástricos1. Revista de Investigación, 6(2), 189-197.

Huelva, R., Garcés, N., Ramos, A., \& León, P. (2004). Evaluación de la bioactividad del humus líquido (Liplant) y sus fracciones húmicas obtenido a partir de vermicompost en el cultivo de soya (Glycine max var. Incasoy24). Programa y Resúmenes. III Taller de Productos Bioactivos. Congreso Científico del Instituto Nacional de Ciencias Agrícolas. La Habana, Cuba. p. 115

Hurtato, D. I., Nocua, S., \& Narváez-Solarte, W. (2012). Valor nutricional de la morera (Morus sp.), matarratón (Gliricidia sepium), pasto india (Panicum máximum) y arboloco (Montanoa quadrangularis) en la alimentación de cuyes (Cavia porcellus). Revista de Veterinária e Zootecnia, 6(1), 56-65.

Manterola, A. H. (2000). La morera, una interesante alternativa forrajera para la ganadería mayor y menor en Chile.

Martín, G., Hernández, I., García, J. C., Sánchez, E., \& Benavides, J. E. (2000). Estudio del efecto de la altura y frecuencia de corte sobre la producción de biomasa en Morera (Morus alba). Memoria IV taller internacional de sistemas silvopastoriles. Estación Experimental de Pasto y Forrajes "Indio Hatuey". Matanzas, Cuba.

Martín, G. J., Noda, Y., Pentón, G., García, D. E., García, F., González, E.,... \& Arece, J. (2007). La morera (Morus alba, Linn.): una especie de interés para la alimentación animal. Pastos y Forrajes, 30, 1-1.

Medina, M. G., Lamela, L., \& García, D. E. (2012). Supervivencia de la Morera (Morus alba) en una asociación sometida a pastoreo y corte. Pastos y Forrajes, 27(3).

Monter, J. V. (2012). Tecnologías alimenticias a base de morera (Morus spp.) en los sistemas de producción animal. Tópicos Selectos de sericultura, 2009, 41.

Noda, Y., Martín, G., \& Mesa, A. R. (2007). Nota técnica: Influencia de la densidad de plantación en el establecimiento de la morera. Pastos y Forrajes, 30 (4), 0-0.

Pentón, G. (2007). Nota Técnica: Comportamiento productivo de la morera sometida a dos alternativas de fertilización orgánica. Pastos y Forrajes, 30 (4), 0-0.

Páez, O. 1991. Cultivo del arroz. Densidad de siembra, control de malezas y fertilización. Fonaiap divulga. No.36.

Okamoto, F., Cunha, E., Bueno, M., Silva, M. D. A., Santos, L., \& Rodrigues, A. D. A. (2008). Desempenho de borregas da raça Santa Inês alimentadas com cana-de-açúcar e ramas de amoreira. Boletim da Indústria Animal, 65(1), 1-6.

Sánchez, M. D. (2002). Mulberry: Un forraje excepcional disponible en casi todo el mundo para la producción animal. Roma: Organización de Alimentos y Agricultura (FAO) de las Naciones Unidas, 271-289.

Toledo, J. M., \& Shultze-Kraft, R. (1982). Metodología para la Evaluación Agronómica de Pastos Tropicales. Manual para la Evaluación Agronómica. Red Internacional de Evaluación de Pastos Tropicales. CIAT, Cali, Colombia, 91-110. 